\title{
Patient and Care Team Perspectives of Telemedicine in Critical Access Hospitals
}

\author{
Meghan M JaKa, PhD', Jennifer M Dinh, MPH'1*, Jeanette Y Ziegenfuss, PhD1, Jerome C Siy, MD², Ameet P Doshi, MD², \\ Yonatan Platt, $\mathrm{MD}^{2}$, John R Dressen, $\mathrm{MHA}^{2}$
}

${ }^{1}$ Center for Evaluation \& Survey Research, HealthPartners Institute, Bloomington, Minnesota; ${ }^{2}$ Hospital Medicine, HealthPartners, Bloomington, Minnesota.

Telemedicine acute care may address issues facing critical access hospitals. This evaluation used web, mail, and telephone surveys to quantitatively and qualitatively assess patient and care team experience with telemedicine in 3 rural critical access hospitals and a large metropolitan tertiary care hospital. Results show that patients, nurses, and clinicians perceived quality of care as high, and they offered feasible recommendations to enhance communication and otherwise improve the experience. Continued work to improve, test, and publish findings on patient and care team experience with telemedicine is critical to providing quality services in often underserved communities. Journal of Hospital Medicine 2020;15:345348. (C) 2020 Society of Hospital Medicine

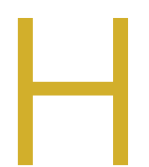
ealthcare delivery in rural America faces unique, growing challenges related to health and emergency care access. ${ }^{1}$ Telemedicine approaches have the potential to increase rural hospitals' ability to deliver efficient emergency care and reduce clinician shortages. ${ }^{2}$ While initial evidence of telemedicine success exists, more quality research is needed to understand telemedicine patient and care team experiences, ${ }^{3}$ especially with real-time, clinician-initiated video conferencing in critical access hospital $(\mathrm{CAH})$ emergency departments (ED). Some experience studies exist, ${ }^{4}$ but results are primarily quantitative ${ }^{5}$ and lack the nuanced qualitative depth needed to understand topics such as satisfaction and communication. ${ }^{6}$ Additionally, few explore combined patient and care team perspectives. ${ }^{5}$ The lack of breadth and depth makes it difficult to provide actionable recommendations for improvements and affects the feasibility of continuing this work and improving telemedicine care quality. To address these gaps, we evaluated a real-time, clinician-initiated video conferencing program with overnight clinicians servicing ED patients in three Midwestern care system $\mathrm{CAHs}$. This evaluation assessed patient and care team (nurse and clinician) experience with telemedicine using quantitative and qualitative survey data analysis.

\section{METHODS}

Because this evaluation was designed to measure and improve program quality in a single healthcare system, it was deemed non-human subjects research by the organization's institution-

*Corresponding Author: Jennifer M Dinh, MPH; Email: Jennifer.m.dinh@ healthpartners.com; Telephone: 952-967-5535.

Published online first May 20, 2020.

Received: November 8, 2019; Revised: March 10, 2020

Accepted: March 10, 2020

(C) 2020 Society of Hospital Medicine DOI 10.12788/jhm.3412 al review board. This brief report follows telemedicine reporting guidelines?

\section{Setting and Telemedicine Program}

This program, designed to reduce the need for on-call hospitalist clinicians to be onsite at CAHs overnight, was implemented in a large Midwestern nonprofit integrated healthcare system with three rural CAHs (combined capacity for 75 inpatient admissions, with full-time onsite ED clinicians and nurses, as well as on-call hospitalist clinicians) and a large metropolitan tertiary-care hospital. All adult patients presenting to CAH EDs between 6 PM and 8 AM were evaluated, as usual, by an onsite ED clinician. If the admitting ED clinician and charge nurse determined that admission was appropriate, patients were signed out to remote hospitalist clinicians and roomed by onsite nurses. Nurses facilitated live audio-video telemedicine "history and physical" visits with remote clinicians via telemedicine carts (AmericanWell C750, Boston, Massachusetts, and ThinkLabs One Electronic Stethoscope, Centennial, Colorado). Alreadyhospitalized patients, as well as patients admitted to a remote clinician, were cared for by the remote clinician and onsite nurse for the remainder of the night, which eliminated the need for local on-call clinicians. The onsite ED clinician responded to emergencies of already-hospitalized patients, but often consulted with remote clinicians to assist virtually with necessary orders and documentation. Remote clinicians were located at the metropolitan tertiary care hospital or home work stations.

Following a pilot period, the full-scale program was implemented in September 2017 and included 14 remote clinicians and 60 onsite nurses.

\section{Survey Administration and Design}

A postimplementation survey was designed to explore patient and care team experience with telemedicine. Patients who received a telemedicine visit between September 2017 and April 
TABLE. Self-Reported Patient and Care Team Experience with the Telemedicine Program

\begin{tabular}{|c|c|c|c|}
\hline & Patients (N = 213) & Clinicians $(N=14)$ & Nurses $(\mathrm{N}=39)$ \\
\hline \multicolumn{4}{|l|}{ Communication } \\
\hline Satisfied with clinician-nurse communication & $92 \%$ & $100 \%$ & $100 \%$ \\
\hline Clinician treated with courtesy and respect & $96 \%$ & NA & NA \\
\hline Clinician explained things in easy-to-understand way & $90 \%$ & NA & NA \\
\hline \multicolumn{4}{|l|}{ Workflow Integration } \\
\hline Satisfied with admission process & NA & $93 \%$ & $100 \%$ \\
\hline Telemedicine improved cross-coverage & NA & $95 \%$ & $95 \%$ \\
\hline \multicolumn{4}{|l|}{ Telemedicine Technology } \\
\hline Technology worked well & $91 \%$ & NA & NA \\
\hline \multicolumn{4}{|l|}{ General Satisfaction } \\
\hline Recommend telemedicine & $85 \%$ & $100 \%$ & $95 \%$ \\
\hline
\end{tabular}

${ }^{1}$ Rated care as a 9 or 10 on a scale of 0-10, where 0 is the worst health care possible and 10 is the best health care possible

2018 were mailed a paper survey. Nonresponders were called by professional interviewers affiliated with the healthcare system. All participating clinicians ( $N=14$, all MDs) and nurses ( $N$ $=60$, all RNs) were emailed an online care team survey with phone-in option. Care team nonresponders were sent up to two reminder emails.

Surveys captured the following five constructs: communication, workflow integration, telemedicine technology, quality of care, and general satisfaction. Existing questionnaires were used where possible; additional items were designed with clinical experts following survey design best practices. ${ }^{8}$ Patient-perceived communication was assessed via three Consumer Assessment of Healthcare Providers and Systems Outpatient and Ambulatory Surgery Survey items. ${ }^{9}$ Five additional program-developed patient survey items included satisfaction with clinician-nurse communication, satisfaction with technology, telemedicine quality of care overall and in comparison with traditional care, and whether or not patients would recommend telemedicine (Table). Four open-ended questions asked patients about improvement opportunities and general satisfaction.

Care team surveys included two items regarding ability to effectively communicate, two about satisfaction with workflow integration, one about technical problems, two about quality of care, and one about general satisfaction. Open-ended questions gathered further information and recommendations to improve communication, workflow integration, technology issues, and general satisfaction.

\section{Analysis}

Closed-ended items were dichotomized (satisfied yes/no); descriptive statistics (frequencies/percents) are presented to quantify patient and care team experience. Quantitative analyses were conducted in SAS software version 9.4 (SAS Institute, Cary, North Carolina). Open-ended responses were coded separately for patient and care team experience, following qualitative content analysis best practices. ${ }^{10}$ A lead coder read all responses, created a coding framework of identified themes, and coded individual responses. A second coder independently coded responses using the same framework. Interrater reliability was calculated for each major theme using percent agreement and prevalence- and bias-adjusted $\mathrm{k}$ (PA$B A K)$ statistic. A single representative quote was selected and lightly edited for each subtheme to deepen understanding and provide respondent voice. ${ }^{11}$

\section{RESULTS}

Of eligible patients mailed a survey ( $\mathrm{N}=408), 3 \%$ self-reported as ineligible, and $54 \%$ completed the survey. This is a maximum response rate (response rate 6 ) according to the American Association for Public Opinion Research. ${ }^{12}$ Patients were 67 years old on average (SD $=15)$, they were primarily white (97\%), and $54 \%$ were female. All clinicians and $63 \%$ of nurses completed the survey. ${ }^{12}$ Clinicians and nurses were $29 \%$ and 95\% female, respectively.

Quantitative results (Table) show generally positive experience across patient and care team respondents. Over $90 \%$ were satisfied with all measures of communication. Care teams had high satisfaction with admissions processes and reported telemedicine improved cross-coverage. Patient-reported technology experience was positive but was less positive from the care team perspective. Care teams reported lower absolute quality of care than did patients but were more likely to per- 
ceive telemedicine as high quality, compared with traditional care. Most patients, clinicians, and nurses would recommend telemedicine.

Qualitatively, four major themes were identified in openended responses with high interrater reliability (PABAK ranging from 0.92 to 0.98 in patient responses and 0.88 to 0.95 in care team responses) and aligned with the quantitative survey constructs: clinician-nurse communication, clinician-patient communication, workflow integration, and telemedicine technology. Patients reported satisfaction with communication with remote clinicians:

"[The clinician] was extremely attentive to me and what was going on. She was articulate and clear. I understood what was going to happen." -Patient

Care teams suggested concrete improvement opportunities:

"I'd prefer to have some time with nursing staff both before and (sometimes) after the patient encounter." -Clinician

"Since we cannot hear what [the clinicians] are hearing with the stethoscope, it's nice when they tell us when to move it to the next spot." -Nurse

Clinicians and nurses gave favorable responses regarding workflow integration, though time (both admissions wait time and session duration) was a reported opportunity:

"It would be helpful if we could speed up the time from admit request to screen time." -Clinician

"When the [clinicians] get swamped, they're hard to get a hold of, and admissions can take a long time. They may have too much on their plates dealing with several locations." -Nurse

Technology issues -internet connection, stethoscope, sound, and screen or camera-were mentioned by patients and care teams, though technology was reviewed favorably overall by most patients:

"I was fascinated by the technology. Visiting someone over a television was impressive. ... The picture, the sound clarity, and the connection itself was flawless." -Patient

Some patients commented that telemedicine was the best option given the situation, but still preferred an in-person doctor:

"If a doctor wasn't available, telemedicine is better than nothing." -Patient
Nurses who would not recommend telemedicine noted the need for personal connection:

"[I] still prefer [an] in-person MD for more personal contact. The older patients often state they wish the doctor would come and see them." -Nurse

Patients who would not recommend telemedicine also desired personal connection:

"I would sooner talk to a person than a machine." Patient

A few clinicians noted the connection with patients would be improved if they knew about others in the room:

"It'd be nice if everyone in the room was introduced. Sometimes people are sitting out of view of the camera and I don't realize they're there until later." -Clinician

\section{CONCLUSION}

These results make important contributions to understanding and improving the telemedicine experience in rural emergency hospital medicine. While the predominantly white patient respondent population limits generalizability, these demographics are representative of the overall population of the participating hospitals. A strength of this evaluation is its contemporaneous consideration of patient and care team experience with both quantitative and rich, qualitative analysis. Patients and care teams alike thought overnight telemedicine was better than the status quo. While our quality of care findings align with some previous literature, ${ }^{13}$ care teams in the current analysis overwhelmingly would recommend telemedicine, whereas some clinicians in prior work would not recommend telemedicine. ${ }^{14}$

In terms of communication, in line with existing literature, some patients still preferred in-person visits, ${ }^{15}$ a view also shared by some care team members. Workflow and technology barriers were raised, corroborating existing work, ${ }^{13}$ but actionable solutions (eg, adding care team-only time before visits or verbalizing when to move stethoscopes) were also identified.

Embedding patient and care team experience surveys and sharing results is critical in advancing telemedicine. Findings from this evaluation strengthen the case for payer reimbursement of telemedicine in rural acute care. Continued work to improve, test, and publish findings on patient and care team experience with telemedicine is critical to providing quality services in often-underserved communities.

\section{Acknowledgments}

The authors would like to acknowledge the contributions of Ann Werner in identifying the patient survey sample, Brian Barklind in identifying source data for the analysis, and both Brian Barklind and Rachael Rivard for conducting the 
analyses and summarizing results. We would also like to thank Kelly Logue for her involvement in conceptualizing the telemedicine evaluation described here, as well as Larisa Polynskaya for her help preparing the manuscript for publication, and the care teams and patients who provided valuable input.

Disclosures: The authors have nothing to disclose.

Funding: Funding for this evaluation was provided by HealthPartners Regions Hospital Foundation (grant \#RG1700315).

\section{References}

1. Nelson R. Will rural community hospitals survive? Am J Nurs. 2017;117(9):1819. https://doi.org/10.1097/01.NAJ.0000524538.11040.7f

2. Ward MM, Merchant KAS, Carter KD, et al. Use of telemedicine for ED physician coverage in critical access hospitals increased after CMS policy clarification. Health Aff. 2018;37(12):2037-2044. https://doi.org/10.1377/ hlthaff.2018.05103

3. AlDossary S, Martin-Khan MG, Bradford NK, Smith AC. A systematic review of the methodologies used to evaluate telemedicine service initiatives in hospital facilities. Int J Med Inf. 2017;97:171-194. https://doi.org/10.1016/j. ijmedinf.2016.10.012.

4. Kuperman EF, Linson EL, Klefstad K, Perry E, Glenn K. The virtual hospitalist: a single-site implementation bringing hospitalist coverage to critical access hospitals. J Hosp Med. 2018;13(11):759-763. https://doi.org/10.12788/jhm.3061.

5. Garcia R, Adelakun OA. A review of patient and provider satisfaction with telemedicine. Paper presented at: Twenty-third Americas Conference on Information Systems; 2017; Boston, Massachusetts.

6. Mair F, Whitten P. Systematic review of studies of patient satisfaction with telemedicine. BMJ. 2000;320(7248):1517-1520. https://doi.org/10.1136/ bmj.320.7248.1517.
7. Khanal S, Burgon J, Leonard S, Griffiths M, Eddowes LA. Recommendations for the improved effectiveness and reporting of telemedicine programs in developing countries: results of a systematic literature review. Telemed $E$ Health. 2015;21(11):903-915. https://doi.org/10.1089/tmj.2014.0194.

8. Fowler Jr FJ. Improving survey questions: Design and evaluation. Vol 38. Thousand Oaks, California: Sage Publications, Inc.; 1995

9. Agency for Healthcare Research and Quality. CAHPS Outpatient and Ambulatory Surgery Survey. https://www.ahrq.gov/cahps/surveys-guidance/oas/ index.html. Accessed August 1, 2017.

10. Ulin PR, Robinson ET, Tolley EE. Qualitative methods in public health: A field guide for applied research. Hoboken, New Jersey: John Wiley \& Sons; 2005.

11. Corden A, Sainsbury R. Using verbatim quotations in reporting qualitative social research: researches' views. York, United Kingdom: University of York; 2006.

12. American Association for Public Opinion Research. Standard Definitions: Final Dispositions of Case Codes and Outcome Rates for Surveys. 2016. https://www.aapor.org/AAPOR_Main/media/publications/Standard-Definitions20169theditionfinal.pdf. Accessed August 1, 2019.

13. Mueller KJ, Potter AJ, MacKinney AC, Ward MM. Lessons from tele-emergency: improving care quality and health outcomes by expanding support for rural care systems. Health Aff. 2014;33(2):228-234. https://doi.org/10.1377/ hlthaff.2013.1016

14. Fairchild R, Kuo SFF, Laws S, O'Brien A, Rahmouni H. Perceptions of rural emergency department providers regarding telehealth-based care: perceived competency, satisfaction with care and Tele-ED patient disposition. Open J Nurs. 2017;7(07):721. https://doi.org/10.4236/ojn.2017.77054.

15. Weatherburn G, Dowie R, Mistry $H$, Young T. An assessment of parental satisfaction with mode of delivery of specialist advice for paediatric cardiology: face-to-face versus videoconference. J Telemed Telecare. 2006;12(suppl 1):57-59. https://doi.org/10.1258/135763306777978560. 